\title{
ENTRE BORUS Y FRAGMENTOS. ANÁLISIS DE UN CONTEXTO ARQUEOMALACOLÓGICO DE PRODUCCIÓN DE CUENTAS EN CERRO COLORADO (SIERRAS DEL NORTE, CÓRDOBA, ARGENTINA)
}

\section{BETWEEN BORUS AND FRAGMENTS. ANALYSIS OF AN ARQUEOMALACOLOGICAL CONTEXT OF BEADS PRODUCTION IN CERRO COLORADO (SIERRAS DEL NORTE, CORDOBA ARGENTINA)}

\author{
Luis Tissera' ${ }^{1}$, Sandra Gordillo², Andrea Recalde ${ }^{3}$ y Sebastián Pastor ${ }^{4}$
}

1Reserva Cultural Natural Cerro Colorado, Dirección de Patrimonio Cultural (ACC).

Calle Pública S/N , Cerro Colorado, Argentina. luistissera@gmail.com ${ }^{2}$ Facultad de Ciencias Físicas, Exactas y Naturales, Universidad Nacional de Córdoba. Centro de investigaciones en Ciencias de la Tierra. CONICET. Av. Vélez Sársfield 1611, X5016CGA, Ciudad Universitaria, Córdoba, Argentina. sandra.gordillo@unc.edu.ar

${ }^{3} \mathrm{CEH} / \mathrm{IEH}$ - CONICET. Universidad Nacional de Córdoba (UNC). Corro 308, Córdoba, Argentina. recaldema@yahoo.com.ar

${ }^{4}$ Centro de Investigación y Transferencia de Catamarca (CITCA-CONICET). S. Fernando del Valle de Catamarca, Argentina.pastorvcp@yahoo.com.ar

Presentado: 22/08/2018 - Aceptado: 10/02/2019

\section{Resumen}

Se realiza el análisis de un conjunto de fragmentos malacológicos pertenecientes al caracol gigante Megalobulimus lorentzianus (Doering 1876) o Borus, procedente de una excavación efectuada en la localidad de Cerro Colorado (provincia de Córdoba). Los resultados obtenidos sugieren que los depósitos estratigráficos de los cuales fueron recuperados estos fragmentos serían de origen antrópico, dado que habría indicios de selección de estos elementos malacológicos. Se discuten estas evidencias en vinculación a la fabricación de cuentas destinadas a la ornamentación personal, a la vez que se propone a los sitios de procedencia de estos hallazgos, como un locus de producción que registra diferentes momentos de una cadena operativa, cuyos componentes se caracterizan por variaciones cuantitativas. En base a la información contextual y a los datos arqueológicos recopilados durante los últimos años en la localidad, por ahora se estima que la cronología para los usos artesanales de este recurso sería compatible con el período Prehispánico tardío propuesto para las Sierras Centrales (ca. 1500-400 AP).

Palabras Clave: cuentas de Borus, locus de producción, cadena operativa, Cerro Colorado.

\footnotetext{
Abstract

An analysis of a set of malacological fragments, belonging to the giant snail Megalobulimus lorentzianus (Doering 1876) or Borus from an excavation carried out in Cerro Colorado (province of Córdoba), is made. The results obtained suggest that the stratigraphic deposits from which these fragments were recovered would be of anthropic origin, since there would be indications of selection of these malacological elements. These evidences are discussed in relation to the manufacture of beads destined to personal ornamentation, while it is proposed
} 
to the sites of origin of these findings, as a locus of production that registers different moments of an operative chain, whose components are characterized by quantitative variations. Based on the contextual information and the archaeological data collected during the last years, it is estimated that the chronology for the artisanal uses of this resource are compatible with the Late Prehispanic period proposed for the Central Sierras (ca. 1500-400 AP).

Key words: Borus beads, locus of production, operational chain, Cerro Colorado.

\section{Introducción}

En las Sierras Centrales son numerosos los aportes que reportan el hallazgo de cuentas de moluscos de origen arqueológico, destinadas a la ornamentación personal (Argüello de Dorsch y Berberián 1985, Berberián 1987, Castellanos 1933, González 1960, Marcellino et al. 1967, Serrano 1945, entre otros). Su utilización también es referida en fuentes documentales del siglo XVI como partes de prendas tejidas, bajo la denominación de "chaquiras" (Bixio y Berberián 1984, Serrano 1945)1․ Estas menciones tempranas, dan cuenta de la importancia de esta materialidad para las comunidades prehispánicas locales.

En general, los contextos de los cuales proceden las cuentas malacológicas se adscriben al final del Holoceno tardío. Sin embargo, algunos hallazgos corresponden a los inicios de ese período, como es el caso de las cuentas identificadas en el denominado Horizonte III de Ongamira (área nororiental del valle de Punilla), que fueron asociadas a grupos cazadores-recolectores (Menghin y González 1954). Dicha estimación cronológica fue confirmada en investigaciones posteriores, que permitieron tanto evaluar su precisión (entre 3515 y 3390 AP), cómo también ampliar la información recuperada (Cattáneo et al. 2013, Yanez et al. 2014). En dicha localidad, además se menciona el hallazgo de tres cuentas de Megalobulimus lorentzianus, procedentes del alero Deodoro Roca (Izeta et al. 2014, Boretto et al. 2018). En tanto, en otro hallazgo documentado en las costas del lago San Roque (sur del valle de Punilla), fueron identificadas 97 cuentas confeccionadas con la misma especie, con un fechado en $3921 \pm 28$ AP (AA108462; cuenta) ${ }^{2}$ (Pastor et al. 2017a). Éstas presentan una tipología peculiar, dada por su gran tamaño y la presencia de un reborde o peristoma, aprovechando así la mayor parte de un sector de la concha del caracol que corresponde a la última vuelta. Su utilización ha sido propuesta como partes de un elemento sonoro (e.g. ideófono de entrechoque) vinculado a algún tipo de atavío ritual. Por último, se menciona el hallazgo de un adorno efectuado sobre la valva perforada de un bivalvo acuático (Anodontites sp., o tal vez Diplodon sp.), procedente de niveles pre-cerámicos del sitio Quebrada del Real 1, en el sector occidental de las Sierras Grandes (Rivero 2015).

No obstante, la presencia de mayor cantidad de cuentas malacológicas se asocia a contextos vinculados con el período Prehispánico tardío o PPT (ca. 1500-400 AP). Hallazgos de este tipo se han registrado tanto en ocupaciones a cielo abierto o campamentos residenciales, como en abrigos rocosos con evidencia de usos transitorios. Las propuestas clásicas los asocian indistintamente a collares o adornos 
(Outes 1911), mientras que otras menciones, procedentes de contextos mejor definidos, sugieren su utilización como partes integrantes de ajuares funerarios en entierros de adultos, generalmente en forma exclusiva (Frenguelli 1921). En esta casuística se destaca el hallazgo de 2.300 cuentas asociadas a la inhumación de un individuo adulto, documentado en el sitio residencial Potrero de Garay, en el valle de Los Reartes (Berberián 1984)3.También se incluye el análisis de 32 cuentas de Borus que componen el ajuar funerario de una mujer adulta, documentado en Villa Santa Rosa, en el valle de Calamuchita (Gordillo y Fabra 2018).

Por otra parte, en la región de Mar Chiquita (llanura oriental), se identificó la especie Anodontites trapesialis que, si bien carece de evidencias de modificaciones antrópicas, integraba una ofrenda mortuoria de un individuo femenino (Fabra et al. 2012).

Otra material malacológico, no disponible en el medio local y que también intervino en la ornamentación personal, corresponde a la utilización de un caracol marino del género Urosalpinx, procedente de la costa Atlántica. Adornos fabricados con estos ejemplares fueron registrados en los sitios arqueológicos de Soconcho, cercano a la localidad de Almafuerte y del lago San Roque, en las porciones australes de los valles de Calamuchita y Punilla, respectivamente (Boman 1920 en Castellanos 1933: 7172, Serrano 1945). Su utilización en la confección de collares como ajuar funerario, también ha sido descripta para los sitios de Laguna Honda, en la cuenca baja del río Tercero y en cercanías de la localidad de Soto (González 1943, Nimo 1946). Procedente de este último se menciona un conjunto compuesto de 34 cuentas, asociado a las vértebras cervicales de un individuo adulto.

En contraposición al amplio consumo de estos artefactos verificado para las Sierras Centrales, la información sobre los contextos de producción de cuentas resulta casi inexistente. En la gruta de Intihuasi, en la vecina provincia de San Luis, González (1960: 154) vinculó la presencia de huellas incipientes de formatización en algunos fragmentos de concha, con la fabricación de adornos personales. Por otra parte, Serrano (1945: 258) menciona para los sitios comprendidos en el actual embalse del lago San Roque, el hallazgo de peristomas completos con callo, formando un anillo cerrado, que asocia a residuos de una industria de adornos a partir de conchas de Borus. Finalmente, para el área serrana de Córdoba la información de otros depósitos sin referencia a contextos de producción -que incluyen escasos fragmentos de conchas o algún ejemplar fragmentado, lo que no descarta un origen tafonómico, se reduce drásticamente (González y Crivelli 1978: 199-200, Pastor 2007: 368).

En este marco, el objetivo general de este trabajo es el análisis de un conjunto de fragmentos de conchas de Borus, procedente de un contexto arqueológico de la localidad de Cerro Colorado. La muestra se evalúa con el objeto de discriminar entre un origen antrópico o natural, considerando posibles indicios de acopio y potenciales pre-formas para cada fragmento. Para tal fin, se sugiere una metodología de análisis adaptada para esta especie, a la vez que se propone presentar evidencia acerca de la 
identificación de un locus de producción de cuentas ornamentales. En lo particular, la información recuperada en dicha localidad permitirá avances significativos para la discusión sobre los procesos involucrados en la manufactura de elementos conquiliológicos en el área de las Sierras Centrales.

\section{Contexto arqueológico de Cerro Colorado}

La localidad arqueológica de Cerro Colorado está emplazada en las Sierras del Norte (Córdoba, centro de Argentina). Su orografía se caracteriza por la distribución irregular de formaciones de areniscas y conglomerados rojizos estratificados, de escasa altitud (inferiores a 1000 m.s.n.m.), asentados de forma discordante sobre el basamento granítico (Painceyra y Martino 2004). La erosión del conjunto sedimentario generó quebradas y valles transversales por donde discurren el río Los Tártagos y algunos colectores subsidiarios (arroyos Los Molles, Aguas de Figueroa, La Quebrada y Las Trancas). El área se encuentra ubicada en un ambiente chaqueño de clima cálido y seco, dominado por especies características de este distrito, como algarrobo (Prosopis spp.), chañar (Geoffrea decorticans) y mistol (Sarcomphalus mistol), donde también convive el mato (Myrcianthes cisplatensis) (Demaio et al. 2002).

Las investigaciones desarrolladas por nuestro equipo en los últimos años, han permitido ampliar las evidencias de ocupación indígena, distribuidas en las márgenes de los cursos de agua y con relación directa a los cerros (Figura 1). Hasta el momento se han documentado cinco sitios residenciales, 29 áreas de molienda de diversos tamaños y 55 sitios con arte rupestre, muchos de estos articulados en una compleja trama de relaciones.
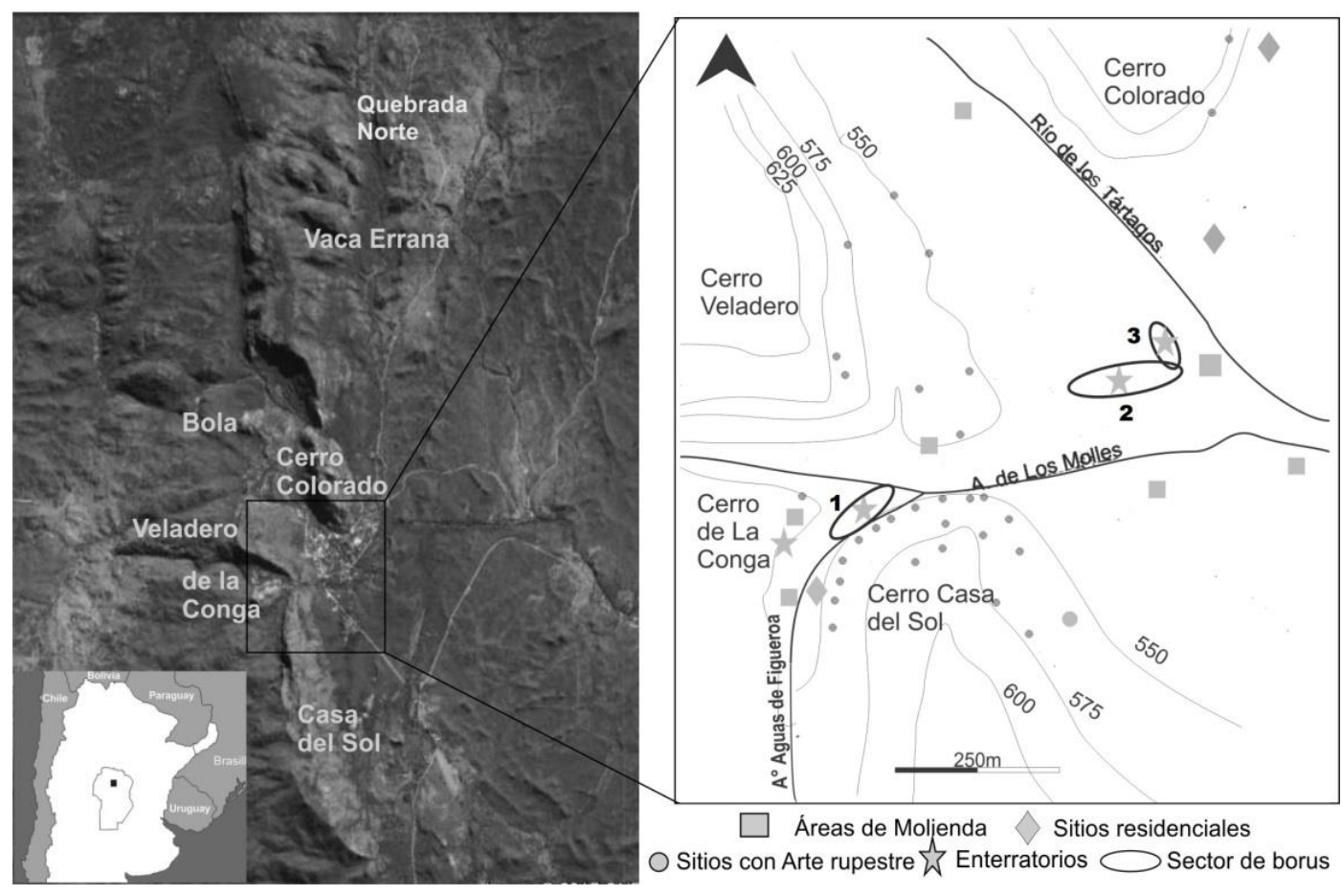

Figura 1: Ubicación de sectores de donde proceden los elementos malacológicos y sitios arqueológicos del entorno. 
En primer lugar, los cinco sitios compatibles con ocupaciones de tipo residencial y asociados a terrazas fluviales potencialmente cultivables, fueron definidos a partir de grandes concentraciones de materiales (líticos, cerámicos, faunísticos y botánicos). Los datos más significativos provienen del análisis de la información arqueobotánica, donde se obtuvo la primera identificación para el área de Sierras Centrales, de los cultígenos quinoa (Chenopodium quinoa) y papa (Solanum tuberosum), además de otras especies frecuentes en el registro arqueológico de esta área como el maíz (Zea mays) (López y Recalde 2016). Los fechados obtenidos en el sitio Quebrada Norte 7 sugieren dos instancias de ocupación adscriptas al PPT, la primera en $1250 \pm 80$ años AP (LP- 3212; carbón) y la segunda en momentos finales de ese período: $405 \pm 21$ años AP (AA 107245; grano de maíz) (Recalde y López 2017).

Las áreas de molienda registradas, generalmente están distribuidas en las márgenes del colector principal y cursos de agua subsidiarios. El análisis de la cantidad, tipo y disposición de instrumentos pasivos ubicados en los soportes rocosos, permite calcular el número factible de operarios que dicha estructura permite acoger de forma simultánea y, de manera indirecta, la esfera de interacción social involucrada en la actividad de molienda (Babot 2004, 2007, Pastor 2015). La conjunción de estas variables indican que en la localidad estarían interactuando tres escalas, una mayoritaria de tipo doméstica ( $\mathrm{N}$ : 15), en la cual intervienen de uno a cuatro individuos, una intermedia, donde se contabiliza la interacción de entre cinco y diez individuos y en la que se promueve la interacción de pequeños grupos familiares (N: 7) y una escala más inclusiva y comunal, que implica la conjunción de más de 10 personas (N: 7), donde se destaca el área de mayor concentración, emplazada en las cercanías del río Los Tártagos, con 40 instrumentos de molienda, la cual está vinculada a los sectores de donde procede la mayor cantidad de fragmentos de Borus identificados (ver más abajo). ${ }^{4}$

Los sitios con arte rupestre, que reúnen cerca de 4.000 motivos pintados y algunos pocos grabados, están emplazados en diferentes sectores de las formaciones que integran esta localidad arqueológica (Colqui 2016, Gardner 1931, González 1940, 1963, Martín de Zurita 1997, Pedersen 1953-1954, Recalde 2015). Los datos recuperados en las tareas de excavación realizadas en cinco aleros rocosos con pinturas, indican que en asociación directa con la ejecución/observación de los motivos rupestres se realizaron otras prácticas, como por ejemplo el procesamiento y consumo de diferentes alimentos, en una densidad y variabilidad de restos menor a la registrada en sitios de carácter residencial. Estas condiciones de ocupación indican un uso probablemente transitorio y poco inclusivo, con reiteraciones en distintos momentos del proceso histórico local. A partir del registro arqueológico procedente de la excavación de uno de estos aleros rupestres, se obtuvo un fechado de $1181 \pm 23$ AP (D-AMS 026995; carbón), lo que sugiere una cronología asignable al PPT, y en consecuencia denota una correspondencia temporal con las ocupaciones de tipo residencial identificadas en el resto de la localidad (Recalde y Colqui 2019). 
En otro orden, con la excepción de la presencia en el museo arqueológico local de algunas puntas de proyectil atribuidas a grupos cazadores-recolectores, correspondientes al Holoceno temprano y medio (e.g. puntas tipo "Ayampitín" y triangulares grandes), no se han identificado hasta el momento en Cerro Colorado, contextos estratigráficos que puedan ser adscriptos a momentos anteriores al PPT.

Finalmente, en el marco de tareas de rescate arqueológico llevadas adelante en la zona, se identificó lo que representa el mayor sitio de funerario de acuerdo a la casuística registrada para la región de las Sierras Centrales (ver Berberián 1984, Fabra et al. 2009, Pastor et al. 2017b). Estudios iniciales han permitido individualizar restos óseos correspondientes a 79 individuos (Díaz com. personal) dispuestos en enterratorios con características diferentes, tanto primarios como secundarios, y posiblemente vinculados a diferentes momentos históricos. El dato a destacar es que dos de los tres sectores de los que proceden los restos malacológicos (Figura 1: Sectores 2 y 3), están directamente vinculados con este espacio de uso comunitario.

\section{El caracol Borus}

Borus es una denominación ampliamente difundida en la literatura y que corresponde al antiguo nombre (Borus oblongus) de la especie actualmente denominada Megalobulimus lorentzianus (Doering 1876). También han sido utilizadas como sinonimias Strophocheilus oblongus y Megalobulimus oblongus. Se trata de un gasterópodo terrestre de gran tamaño, con una amplia área de distribución que se extiende desde el sur de Bolivia hasta la Provincia de Córdoba en Argentina (Beltramino 2014).

Esta especie se caracteriza por un caparazón de pared gruesa y sólida, de forma oval oblonga. Su coloración es blanquecina opaca; el reborde de la abertura o peristoma es de una coloración intensa, rosado purpúrea; y el ombligo está parcialmente oculto por el extremo columelar y el callo. En un trabajo reciente Gordillo (2018) brinda mayor información sobre este molusco, como así también las razones de interés multidisciplinario que ameritan que sea reconocido como una especie biocultural y de interés local para la provincia de Córdoba.

\section{Material y método}

El material de estudio está constituido por un conjunto de fragmentos de Borus procedentes de la localidad arqueológica de Cerro Colorado. Se consideraron además los fragmentos con signos de formatización, que fueron discriminados en pre-formas y cuentas.

La aproximación sistemática para su identificación se basó en la presencia de conchas enteras resguardadas en el museo local. Las mismas, representadas por 16 ejemplares adultos y dos juveniles, conforman el material de referencia para la caracterización tipológica de los fragmentos, aunque no fueron incluidas en los análisis. 
Respecto a los fragmentos, los elementos diagnósticos en relación a la especie considerada fueron: su tamaño, textura, coloración y la presencia de la espira, sector umbilical de la columela o una fracción de peristoma. Con la finalidad de estimar la frecuencia de cada tipo, estos fragmentos fueron tipificados, resultando siete categorías, según la pertenencia de cada elemento a un sector diferente de la concha (Tabla 1, Figura 2). A partir de dicha tipificación se pudieron cuantificar los fragmentos que reúnen las características morfológicas y las medidas mínimas para conformar una pre-forma.

\begin{tabular}{ll}
\hline Tipología & \multicolumn{1}{c}{ Descripción del material } \\
\hline $\mathrm{A}$ & Espira (Fig. 1 A) \\
$\mathrm{B}$ & Tracto umbilical de la columela (Fig. 1 B) \\
$\mathrm{C}$ & Callo y peristoma formando un anillo (Fig. 1 C) \\
$\mathrm{D}$ & Peristoma con última vuelta (Fig. 1 D) \\
$\mathrm{E}$ & Peristoma solo o con pequeña fracción de última vuelta (Fig. 1 E) \\
$\mathrm{F}$ & Fragmento de vuelta con reborde de sutura (Fig. 1 F) \\
$\mathrm{G}$ & Fragmento de vuelta sin reborde de sutura (Fig. 1 G) \\
\hline
\end{tabular}

Tabla 1. Tipologías de fragmentación de Borus

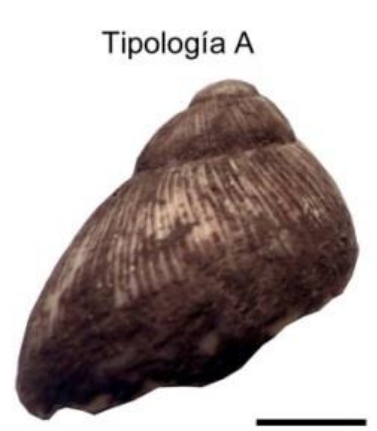

Tipología D
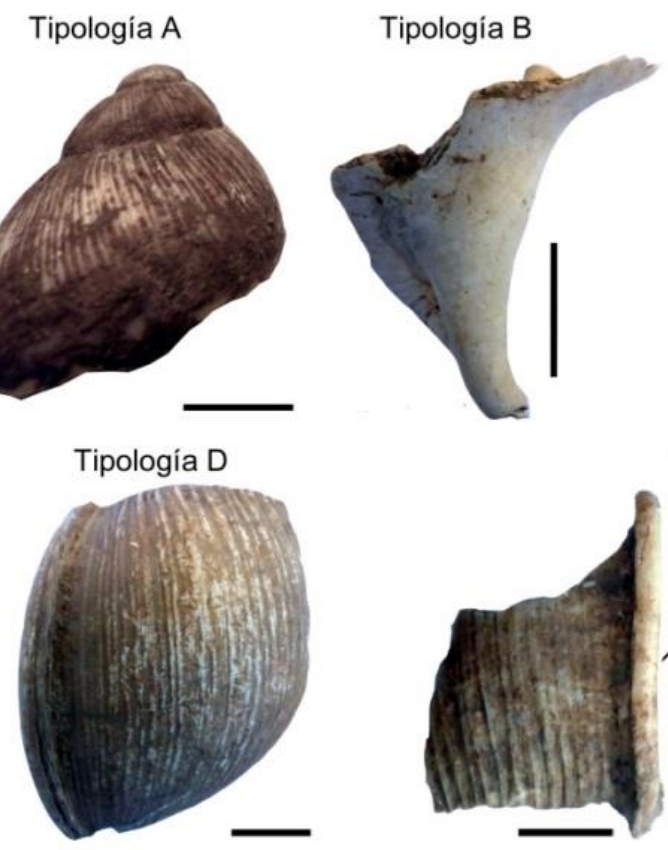

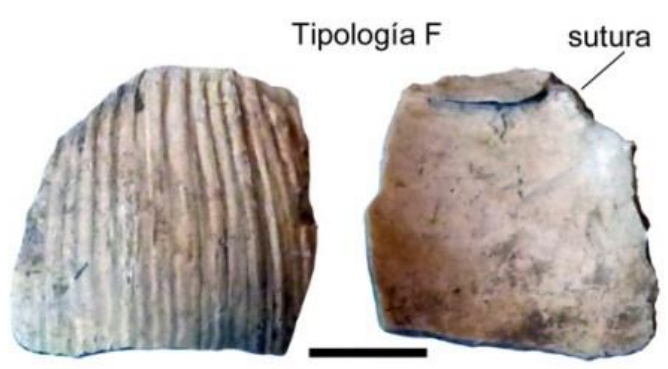

Tipología E

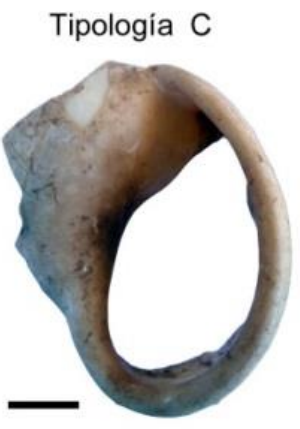

peristoma
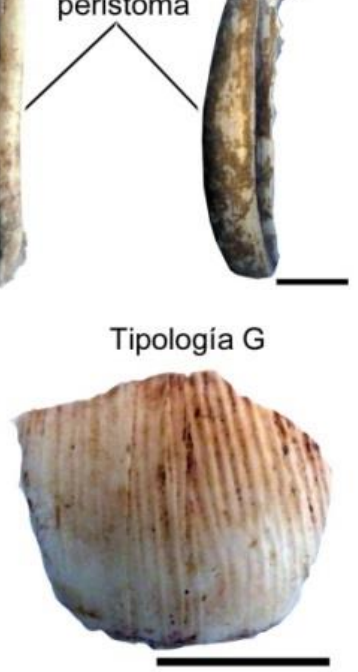

Figura 2. Tipologías de fragmentación de Borus 


\begin{tabular}{|c|l|c|c|c|l|c|}
\hline $\mathbf{N}^{\circ}$ & \multicolumn{1}{|c|}{ Forma } & Perforación & $\boldsymbol{\varnothing}$ max. & $\boldsymbol{\varnothing}$ orificio & \multicolumn{1}{c|}{ Observaciones } & Morfoespecie \\
\hline 1 & Sub-rectangular & Ausente & 19 & -- & Preforma, contorno pulido, no perforada & Megalobulimus sp \\
\hline 2 & Irregular & Cónica interna & 17 & 3 & Preforma con peristoma, contorno no pulido & Megalobulimus sp \\
\hline 3 & Rectangular & Bicónica & 12 & 2,2 & Cuenta con peristoma & Megalobulimus sp \\
\hline 4 & $\begin{array}{l}\text { Sub- } \\
\text { cuadrangular }\end{array}$ & Cónica interna & 18 & 3 & Cuenta con peristoma & Megalobulimus sp \\
\hline 5 & Cuadrangular & Bicónica & 13 & 2,5 & Cuenta con peristoma & Megalobulimus sp \\
\hline 6 & Sub-oval & Bicónica & 27 & 2,5 & Cuenta grande, perforación subcircular & Megalobulimus sp \\
\hline 7 & Circular & Cónica interna & 10 & 1,5 & & Megalobulimus sp \\
\hline 8 & Circular & Cilíndrica & 6,5 & 1,8 & Cuenta con superficie pulida, opaca & No identificada \\
\hline 9 & Circular & Cónica externa & 9,5 & 3 & Cuenta semi-pulida & Megalobulimus sp \\
\hline 10 & Sub-circular & Cónica interna & 10,5 & 3 & Cuenta semi-pulida con reborde & Megalobulimus sp \\
\hline 11 & Circular & Bicónica & 6 & 1,8 & & Megalobulimus sp \\
\hline 12 & Circular & Cónica interna & 8,5 & 2 & & Megalobulimus sp \\
\hline 13 & Circular & Cónica interna & 7 & 1,5 & & Megalobulimus sp \\
\hline 14 & $\begin{array}{l}\text { Sub- } \\
\text { cuadrangular }\end{array}$ & Cónica interna & 14 & S/D & Cuenta rota, con un lado iridiscente & Unionidae \\
\hline 15 & Circular & Cónica interna & 13 & 2 & Cuenta con un lado iridiscente & Unionidae \\
\hline 16 & Circular & Cónica interna & 12 & 2,5 & Cuenta semi-pulida & Megalobulimus sp \\
\hline
\end{tabular}

Tabla 2: Tipificación de preformas (1-2) y cuentas (3-16) de Cerro Colorado 
Con relación a las cuentas, se consideró su tamaño, forma, presencia de orificio y posición (Tabla 2, Figura 3). La ausencia del orificio en fragmentos regularizados perimetralmente, o bien, su presencia en fragmentos de forma irregular, fueron los atributos utilizados para diferenciar una cuenta de una pre-forma ${ }^{5}$.

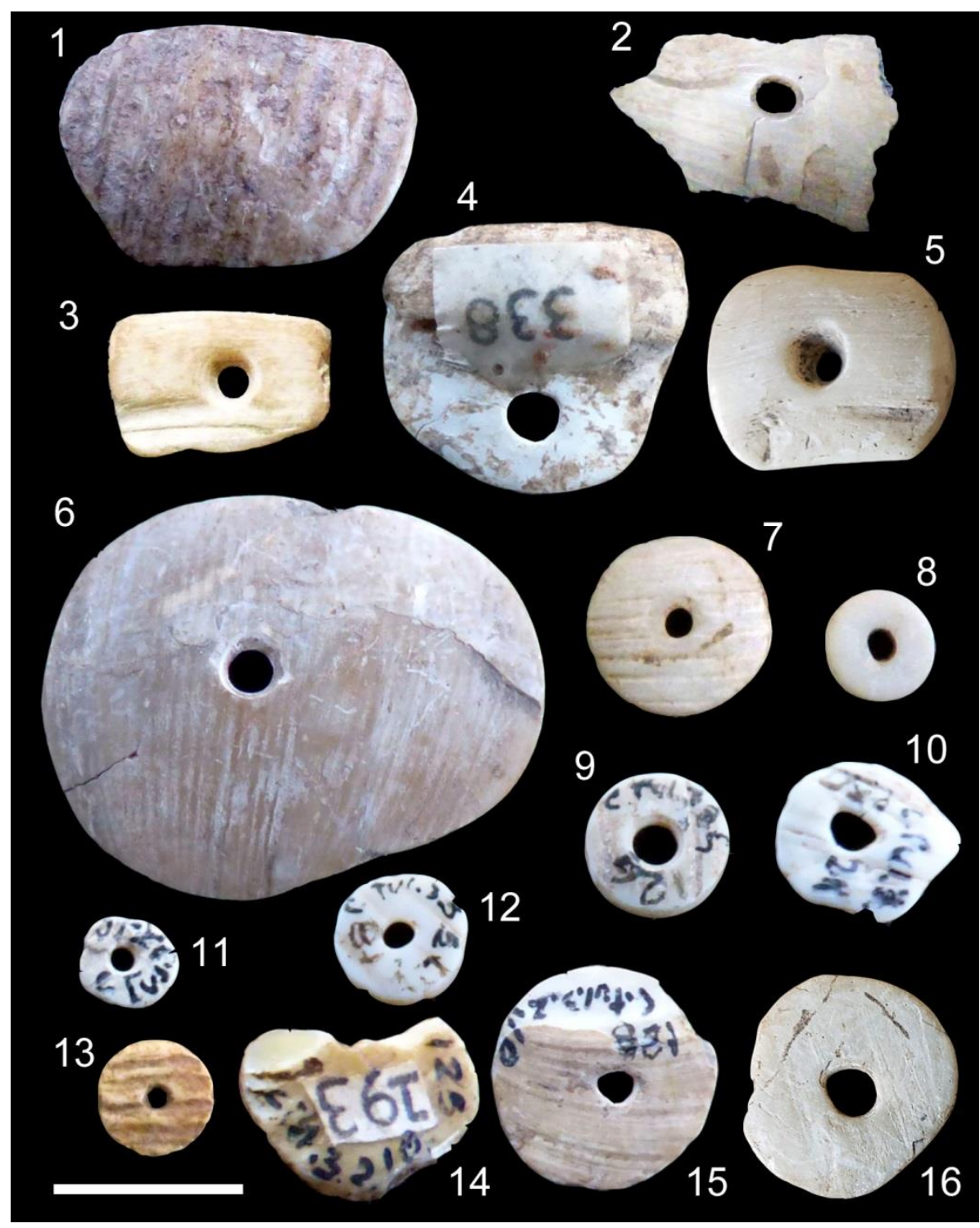

Figura 3. Cuentas y preformas de Cerro Colorado

A los fines de estimar si un conjunto de fragmentos de Borus, recuperados de un mismo nivel estratigráfico, tiene un origen tafonómico o es producto de roturas antrópicas intencionales, se propone una metodología de análisis aplicada a este taxón específico. En primera instancia se procedió a identificar la presencia de fragmentos diagnósticos que permitan contabilizar individuos. Estos incluyen la espira (tipología A; Figura 3A), el sector umbilical, en la base de la columela (tipología B; Figura 3B) o el peristoma entero (tipología C; Figura 3C). 
En los casos en que se observó más de un fragmento de peristoma, se midieron y compararon las medidas del ancho. Este análisis se basa en que la medida del peristoma para un mismo ejemplar, se mantiene constante o presenta variaciones poco significativas. Esto permite asociar fragmentos de peristomas procedentes del mismo nivel estratigráfico, cuyos anchos son iguales o muy similares, a un único individuo. De manera opuesta, fragmentos de peristomas procedentes de un mismo nivel estratigráfico con diferentes medidas del ancho, pueden ser asignados a distintos individuos.

A partir de estas observaciones, la ausencia o la baja correspondencia de componentes diagnósticos en un conjunto de fragmentos (e.g. presencia de fragmentos de peristomas de diferentes individuos y ausencia de espiras y/o sectores umbilicales), pueden considerarse como indicios que permiten descartar roturas tafonómicas in situ o posdeposicionales.

\section{Procedencia del material}

A diferencia de la mayor dispersión y densidad que alcanzan los restos culturales identificados en el área arqueológica de Cerro Colorado, los materiales malacológicos proceden en su totalidad de una zona de la localidad, circunscripta a tres sectores de escasas dimensiones (Figura 1). Según los inventarios del museo local y la información arqueológica, 68 fragmentos asignables a Borus ${ }^{6}$ provienen de un sector localizado en las inmediaciones de la juntura de los arroyos Aguas de Figueroa y Los Molles, donde se documentaron cinco sitios arqueológicos (Sector 1, Figura 1). Uno de estos sitios es un abrigo pintado denominado El Español a Caballo (CS4), en tanto que otro sitio corresponde a otro abrigo denominado Casa de los Peones (CdlC 2) que no presenta evidencias de arte rupestre. Ambos abrigos fueron excavados por González en 1963 y sus resultados permanecen inéditos. Además de los fragmentos recuperados en ambos abrigos, se registró el hallazgo de algunas cuentas malacológicas procedentes de sus perfiles estratigráficos.

Otro conjunto reúne 712 fragmentos también asignables a Borus, que corresponden a sondeos y excavaciones realizados recientemente por nuestro equipo de trabajo, como por Delprato $(1974-83,1983)$ a fines del siglo pasado. Este conjunto proviene de dos sectores contiguos localizados sobre una terraza fluvial colindante con el río Los Tártagos (sectores 2 y 3, Figura 1). Los sectores mencionados también reportaron la mayoría de las cuentas y la totalidad de las pre-formas documentadas en la localidad.

Para el presente análisis se consideraron 380 fragmentos que provienen de este último conjunto y que corresponden a una excavación sistemática (L-Cha.1: Sector 3. Figura 1). El área intervenida abarcó $2 \mathrm{~m}^{2}$ de superficie por un metro de profundidad. El criterio para esta selección se basó en que esta muestra representa el material de mayor control, ya que no conocemos las metodologías llevadas adelante en las otras intervenciones. 
Los datos proporcionados resultan significativos dado que, en los trabajos de excavación realizados en espacios residenciales y en otros sitios de ocupación temporaria (e.g. abrigos rocosos) ubicados en diferentes sectores de la localidad, no se han reportado cuentas, fragmentos, ni conchas enteras de Borus.

\section{Resultados}

Tipificación de cuentas y pre-formas

Entre los materiales malacológicos formales, se registraron catorce cuentas, de las cuales doce son asignables a la especie de origen local M. lorentzianus (Figura 3: 3 al 7, de 9 al 13 y 16), dos cuentas iridiscentes que podrían corresponder a alguna especie exótica de bivalvo dulceacuícola (Anodontites sp. o Diplodon sp.) (Figura 3: 14 y 15) y una cuenta no identificada (Figura 3: 8) debido a la ausencia de rasgos diagnósticos producto de modificaciones antrópicas. Respecto a las cuentas de material exótico, ambas especies ocupan actualmente la cuenca Parano-Platense, en tanto que la primera pudo tener otro vector como la cuenca del río Tercero, donde hoy se halla extinta (Corigliano et al. 1996; Rumi et al. 2008). La importancia de estos hallazgos, aunque escapa a los objetivos de este trabajo, es señalar la existencia de redes de interacción entre comunidades distantes.

La morfología de estos adornos es variable, aunque predominan las formas discoidales, y las técnicas de manufactura evidencian distintos tratamientos, observados en la dirección del orificio y en la parte externa de la concha, donde algunas cuentas han sido modificadas por pulimento (Tabla 2).

Entre las pre-formas se agregan tres fragmentos de concha sub-circulares sin perforar (una de estas en Figura 3: 1) y un fragmento de bordes irregulares perforado (Figura 3: 2), todas atribuibles a Borus. La existencia de una pre-forma de contorno irregular perforada y otras de contornos regularizados sin perforar, estaría indicando que no hay un orden rígido en los pasos que conforman la cadena operativa, respecto a la regularización perimetral y perforación de los fragmentos seleccionados para elaborar la cuenta.

Análisis de fragmentos

Dentro de los grupos tipológicos considerados para la muestra (N: 380) (Figura 2), los fragmentos con las tipologías $\mathrm{F}$ y $\mathrm{G}$ fueron los más representados, ya que reúnen el $90,51 \%$ (con un $19,73 \%$ y 70,78\%, respectivamente). Fragmentos con peristoma que corresponden a la tipología E representan el 6,31\% y con tipología D, el 0,265\% de la muestra.

La tipología B (sector umbilical) representa sólo el 2,89\% de la muestra, siendo el principal tipo de fragmento hallado que permite contabilizar individuos ya que no se registraron fragmentos representados por las tipologías A (espira), ni C (peristoma formando un anillo) (Tabla 3). 


\begin{tabular}{|c|c|c|c|c|c|c|c|c|c|}
\hline NIVEL & A & B & C & D & E & F & G & Cuenta & TOTAL \\
\hline N1 & - & 1 & - & - & - & 3 & 10 & - & 14 \\
\hline N2 & - & - & - & - & 2 & 3 & 16 & - & 21 \\
\hline N3 & - & - & - & - & 2 & 4 & 16 & 1 & 23 \\
\hline N4 & - & - & - & - & - & 1 & 2 & - & 3 \\
\hline N5 & - & 2 & - & 1 & 6 & 10 & 28 & - & 47 \\
\hline N6 & - & 1 & - & - & 3 & 7 & 31 & - & 42 \\
\hline N7 & - & 1 & - & - & 1 & 14 & 40 & - & 56 \\
\hline N8 & - & 2 & - & - & 1 & 9 & 34 & - & 46 \\
\hline N9 & - & - & - & - & 1 & 5 & 33 & - & 39 \\
\hline N10 & - & 1 & - & - & 3 & 9 & 24 & - & 37 \\
\hline N11 & - & 3 & - & - & 4 & 6 & 23 & - & 36 \\
\hline N12 & - & - & - & - & 1 & 4 & 12 & - & 17 \\
\hline TOTAL & $\mathbf{0}$ & $\mathbf{1 1}$ & $\mathbf{0}$ & $\mathbf{1}$ & $\mathbf{2 4}$ & $\mathbf{7 5}$ & $\mathbf{2 6 9}$ & $\mathbf{1}$ & $\mathbf{3 8 1}$ \\
\hline
\end{tabular}

Tabla 3: Tipología de los fragmentos de Borus identificados en la estratigrafía del sitio L-Cha.1

Estos resultados estarían indicando que hay una subrepresentación de algunos componentes como aquellos que se corresponden a las tipologías $\mathrm{A}, \mathrm{B}$ y $\mathrm{C}$, lo que no ocurriría en condiciones de roturas naturales.

En los casos de fragmentos con peristomas (tipologías D y E) hallados en los niveles 2, 5, 6, 10 y 11, se registran medidas dispares en cada nivel, lo que denota que no se corresponden a fragmentos de un mismo individuo. La cuantificación y medición de estos componentes indican la presencia de entre dos y hasta cinco individuos por nivel, a lo que se suma la ausencia de espiras (tipología A) en todos los casos y una baja o nula correspondencia con los sectores umbilicales (tipología B). A su vez, en los niveles 3, 9 y 12 con la presencia de fragmentos con peristoma (tipología E) de al menos un individuo, no se observan fragmentos con tipología $\mathrm{B}$, en tanto que en los niveles 1 y 8 , los fragmentos con peristomas resultan ausentes 0 sub-representados respecto a aquellos con sector umbilical. En definitiva, se observa una mayor selección de algunas tipologías (tipologías F, G y en menor proporción E y D), y sub-representación (tipología B) y ausencia de otras (tipología A y C).

De este modo, los datos obtenidos para una misma unidad estratigráfica, dan cuenta de la presencia de fragmentos pertenecientes a distintos individuos, entre los cuales la ausencia de otras partes de la concha impide remontar ejemplares completos, lo que pone en duda la posibilidad de roturas tafonómicas por causas naturales.

En otro orden, a partir del análisis de la variedad de material conocido, con base en referencias bibliográficas y al relevamiento de cuentas que integran distintas colecciones museísticas de la provincia de Córdoba, el $81,50 \%$ de los fragmentos 
analizados integra un rango compatible con las medidas necesarias para producir cuentas ornamentales, de modo que podrían considerarse como potenciales preformas.

Estos resultados sugieren que los fragmentos analizados serían producto de actividades intencionales, vinculadas a tareas de manufactura y tal vez, al acopio de material malacológico. Según nuestra interpretación, los ejemplares completos habrían sido fracturados en otra locación y determinadas partes de las conchas depositadas en el lugar del hallazgo (e.g. acopio de material), o bien, si las fracturas se realizaron in situ, las partes faltantes fueron retiradas, producto de acciones intencionales de carácter antrópico.

\section{Discusión}

Los fragmentos analizados representan al caracol terrestre Megalobulimus lorentzianus, especie que actualmente no habita en la localidad de Cerro Colorado, aunque si en áreas vecinas. Fuera de los sitios arqueológicos de donde proviene el material malacológico, se advierte la presencia de especímenes completos en distintos perfiles que conforman las antiguas terrazas fluviales, donde se muestran insertos en los sedimentos loéssicos del Cuaternario. La ausencia de coloración que denota el peristoma de estos ejemplares, indica que se trata de especímenes no actuales (Martínez Soler 1958-59:274). En concordancia con esta evidencia, un ejemplar de este gasterópodo hallado en un perfil sobre la costa del arroyo La Quebrada, fue fechado en $6640 \pm 100$ AP (LP-409; concha; Strelin 1995). Si bien la región norte de Córdoba actualmente es hábitat de esta especie, se ignora en qué momento se produjo su extinción local (Gordillo 2018).

Las excavaciones y prospecciones realizadas en los tres sectores de la localidad donde fueron identificados los elementos malacológicos, dan cuenta de otros vestigios arqueológicos que actúan como indicadores de prácticas sociales específicas. En la excavación del sitio L-Ch.1, junto al material de origen malacológico, se documentaron restos de fauna (artiodáctilos, placas de Euphractinae y cáscaras de huevos de Rheidae, entre otros), acompañados de fragmentos cerámicos, líticos y algunos macrorrestos vegetales, actualmente en proceso de análisis. Este sitio se articula con otras áreas del sector que sugieren una interacción social que supera el ámbito doméstico, como la infraestructura de molienda y el espacio funerario. En este sentido, dichos sectores pueden ser definidos como espacios públicos, construidos a partir de la reiteración de otras prácticas sociales como la inhumación y la molienda colectiva, los cuales, de algún modo habrían incorporado en determinado momento histórico de su trayectoria, un conjunto de actividades vinculadas al procesamiento de las conchas de Borus.

Cabe recordar que este gasterópodo representó para las sociedades precolombinas del noroeste de Argentina un elemento de gran valor simbólico, donde resulta frecuente su presencia como acompañamiento de los difuntos en tumbas y urnas funerarias (Boman 1908; De Feo 2012; Nordenskiöld 1903; Ortiz y Vargas 2015). En la provincia de Córdoba, Frenguelli (1921) menciona su hallazgo asociado a esqueletos 
humanos en las costas del lago San Roque, en tanto que un registro de estas características, vinculado a otra especie de molusco (Anodontites trapesialis), también se reportó en las costas de la laguna Mar Chiquita (Fabra et al. 2012). Sin embargo, en los sectores que integran este análisis no se han documentado hasta el momento contextos claros de asociación entre entierros y Borus, aunque ambos comparten vínculos de espacialidad, lo que amerita profundizar el estudio de estos elementos en su conjunto.

Respecto a la totalidad de los elementos malacológicos documentados en la localidad, integran todos los estadios de una cadena operativa para la confección de cuentas y/o adornos de Borus. Los ejemplares completos y fragmentos amorfos están mayormente representados ( $\mathrm{N}: 780)$, mientras que cuentas y pre-formas son escasas $(\mathrm{N}$ : 13). Esta representación diferencial, donde el acopio del material y su descarte involucran mayoritariamente a fragmentos correspondientes a distintas partes de la concha y en menor medida a ejemplares completos, podría indicar dos situaciones probables. La primera, es que en el área investigada se haya realizado principalmente la reducción de conchas de Borus, en tanto que su acabado final podría haber ocurrido en otra locación, posibilidad que no excluye la manufactura de algunas cuentas y experimentos in situ. Otra posibilidad es que la escasa presencia de cuentas terminadas podría ser compatible con una producción destinada a un consumo extra-local. Esto implicaría considerar una escala mayor para la circulación de estos artefactos, y una producción diferenciada a partir de determinados locus que actuaron como proveedores dentro de esos circuitos, tema cuyo abordaje requiere ampliar en el futuro las líneas planteadas en esta investigación.

\section{Consideraciones finales}

Si bien, el hallazgo de cuentas de la especie Megalobulimus lorentzianus resulta frecuente en el área de las Sierras Centrales, los escasos contextos vinculados a su producción no resultan claros. La presencia de numerosos fragmentos de este molusco, hallados en contextos arqueológicos de Cerro Colorado, representa la mayor densidad documentada en dicha área para este recurso.

El análisis de un conjunto de fragmentos recuperados a partir de la excavación del sitio L-Cha.1, de donde procede la muestra considerada, sugiere un origen antrópico para la formación de los depósitos conquiliológicos.

Con base en la metodología aplicada para este taxón específico, los resultados basados en la presencia/ausencia, cuantificación y correspondencia de partes diagnósticas, sumado a la variabilidad de elementos arqueomalacológicos, se consideran indicadores de actividades vinculadas a la manufactura de conchas de Borus.

A una escala mayor, los resultados de las investigaciones arqueológicas en Cerro Colorado, hasta el momento sugieren que esta actividad, destinada a la fabricación de adornos personales, no implicó una práctica extendida espacialmente, sino que se 
restringió a sectores circunscriptos de la localidad, los que constituyeron lugares de uso público construidos a partir de otras prácticas sociales como la inhumación de cuerpos y la molienda colectiva.

Agradecimientos: A Héctor Biurrún, Marina Martínez y Cintia Cuestas, por su colaboración en los trabajos de campo. Al señor Carlos Saavedra, por facilitar el acceso al sitio L-Cha.1 de su propiedad.

\section{Notas}

${ }^{1}$ La Relación Anónima señala que "las camisetas que traen vestidas son hechas de lana y tejidas primeramente con chaquiras a manera de malla menuda de muchas labores en las aberturas y ruedos y bocamangas" (Levillier, 1930: 321; citado en Bixio y Berberián 1984).

2Estudios recientes dan cuenta de la confiabilidad de los fechados realizados sobre valvas de moluscos terrestres del género Megalobulimus ya que muestran las variaciones isotópicas del carbono atmosférico (Macario et al. 2016).

${ }^{3}$ No obstante, entre las prácticas mortuorias predomina la ausencia de ajuares tal como analizan Fabra et al. (2009) para 19 casos distribuidos en diferentes regiones serranas y en Mar Chiquita.

${ }^{4} \mathrm{La}$ infraestructura de molienda incluye dos soportes rocosos. El de mayor tamaño expone un corte central, a través del cual se construyó una calle para el tránsito vehicular, lo que provocó una importante pérdida de artefactos pasivos de molienda, estimado en no menor a 20 unidades adicionales

${ }_{5}^{5}$ n ejemplar de fragmento amorfo perforado fue también considerado como preforma.

${ }^{6}$ Este corpus incluye fragmentos provenientes de rescates realizados como mitigación, ante intervenciones no arqueológicas que afectaron los depósitos estratigráficos (cavado de zanjas para infraestructura urbanística) y que se han conservado junto a otros materiales procedentes de la misma ubicación, en lotes que fueron embolsados in situ y que hasta ahora no habían sido limpiados ni clasificados.

\section{Bibliografía citada}

Argüello de Dorsch, E. y E. E. Berberián

1985 Investigaciones arqueológicas en el yacimiento La Playa (Departamento Punilla, Córdoba). Comechingonia 5: 137-151.

Babot, M. P.

2004 Tecnología y utilización de artefactos de molienda en el Noroeste prehispánico. Facultad de Ciencias Naturales e IML, Universidad Nacional de Tucumán, Argentina, 303 pp. Tesis Doctoral, San Miguel de Tucumán.

2007 Organización social de la práctica de molienda: casos actuales y prehistóricos del Noroeste Argentino. En Procesos sociales prehispánicos en el sur andino: la vivienda, la comunidad y el territorio, A. E. Nielsen, M. Rivolta, V. Seldes, M. Vázquez y P. Mercolli (eds), pp. 259-29. Brujas, Córdoba. 
Beltramino, A. A.

2014 Distribución histórica y área de distribución potencial del megamolusco terrestre Megalobulimus lorentzianus (Doering, 1876) (Gastropoda: Pulmonata) en América del Sur. Boletín de la Asociación Argentina de Malacología 4(1): 10-13.

Berberián, E. E.

1984 Potrero de Garay: una entidad sociocultural tardía de la región serrana de la provincia de Córdoba (República Argentina). Comechingonia 4: 71-138.

1987 Crónicas del Tucumán. Siglo XVI. Conquistadores de Indias I. Comechingonia Revista de Antropología e Historia, Córdoba.

Bixio, B. y E. E. Berberián

1984 Etnohistoria de la región de Potrero de Garay. Comechingonia 3(2): 11-46.

Boman, E.

1908 Antiquités de la región andine de la Republique Argentine et du Désert d'Atacama. Imprimerie Nationale, Paris.

Boretto, G. M., Gordillo, S., Izeta A. D., Colombo, F., Martinelli, M. y G. R. Cattáneo 2018 Cuentas ornamentales en un contexto de cazadores-recolectores de la provincia de Córdoba: análisis mineralógico y microestructural de la concha de "Borus". Arqueología 24(1): 213-223.

Castellanos, A.

1933 El hombre prehistórico de la provincia de Córdoba (Argentina). Revista de la Sociedad Amigos de la Arqueología 8: 5-88.

Cattáneo. G.R.; Izeta, A. D. y M. Takigami

2013 Primeros fechados radiocarbónicos para el sector B del sitio Alero Deodoro Roca (Ongamira, Córdoba, Argentina). Relaciones de la Sociedad Argentina de Antropología 38(2): 1-9.

Colqui, E.

2016 Primeras aproximaciones al análisis del arte rupestre de Quebrada Norte en el contexto de Cerro Colorado (sierras del Norte, Córdoba). La Zaranda de Ideas 14(2): 73-92.

Corigliano, M. C., C. M. Gualdoni, A. M. Oberto y G. B. Raffaini

1996 Macroinvertebrados acuáticos de Córdoba. En Biodiversidad de la Provincia de Córdoba, volúmen I, I. E. Di Tala y E. H Bucher (eds), pp. 119-165. Universidad Nacional de Río Cuarto.

De Feo, M. E.

2012 Prácticas funerarias en el sitio formativo tardío Tres Cruces 1, Quebrada del Toro, Salta, Argentina (Siglos V al X D.C.). Relaciones de la Sociedad Argentina de Antropología 37(1): 43-64.

Delprato, J.

1983 Informe de excavación $\mathrm{N}^{\circ} 2$ (21/2/83). Dirección de Patrimonio de la Provincia de Córdoba, Agencia Córdoba Cultura. Manuscrito inédito.

1974-83 Catálogo de elementos arqueológicos de Cerro Colorado. Parque Arqueológico y Natural de Cerro Colorado, Córdoba. Manuscrito inédito. 
Demaio, P., Karlin, U. O. y M. Medina

2002 Árboles nativos del centro de la Argentina. Literature of Latin America, Buenos Aires.

Doering, A.

1876 Apuntes sobre la fauna de moluscos de la República Argentina (Tercera Parte). Boletín de la Academia de Ciencias Exactas 2: 336-337.

Fabra, M., Salega, M. S. y C. V. González

2009 Comportamiento mortuorio en poblaciones prehispánicas de la región austral de las sierras Pampeanas durante el Holoceno. Arqueología 15: 165-188.

Fabra, M., Gordillo S. y E. L. Piovano

2012 Arqueomalacología en las costas de Ansenuza: análisis de una almeja nacarífera (Anodontites trapesialis) hallada en contexto funerario del sitio El Diquecito (laguna Mar Chiquita, Córdoba). Arqueología 18: 257-266.

Frenguelli, J.

1921 Conchas de "Borus" en los paraderos indígenas del río San Roque (Sierra Chica de Córdoba, Departamento de Punilla). Boletín de la Academia Nacional de Ciencias de Córdoba 26: 404-418.

Gardner, G. A.

1931 Rock-paintings of North West Córdoba. Calderon Press, Oxford.

González, A.R.

1940 Las pinturas rupestres del Cerro Colorado (Provincia de Córdoba). Revista Geográfica Americana 86(14): 333-336.

1943 Paradero indígena de Soto. Anales del Museo Argentino de Ciencias Naturales "Bernardino Rivadavia" 84.

1960 La estratigrafía de la gruta de Intihuasi (Provincia de San Luis, República Argentina) y sus relaciones con otros sitios pre-cerámicos de Sudamérica. Revista del Instituto de Antropología 1: 1-290.

1963 Las pinturas indígenas del Cerro Colorado. Revista Gacetika 63: 14-19.

González, S. y E., Crivelli.

1978 Excavaciones arqueológicas en el abrigo de Los Chelcos (Departamento San Alberto, Córdoba). Relaciones de la Sociedad Argentina de Antropología 12: 183-206.

Gordillo, S.

2018 El caracol gigante Megalobulimus lorentzianus (Doering, 1876): una especie biocultural de la provincia de Córdoba. Revista de la Facultad de Ciencias Exactas, Físicas y Naturales 5 (2): 63-69.

Gordillo, S. y M., Fabra.

2018 Cuentas malacológicas asociadas a restos óseos humanos en el Holoceno tardío de la región central de Argentina. Revista del Museo de Antropología (11)2: 49-58.

Izeta, A.D., Gordillo, S., Costa, T.S. y A. Robledo

2014 Los gasterópodos del sector B del sitio alero Deodoro Roca, Valle de Ongamira (Córdoba, Argentina): un análisis preliminar. Revista Chilena de Antropología 29: 74-80. 
López, L. y M. A. Recalde

2016 The first quinoa (Chenopodium quinoa wild) macrobotanical remains at Sierras del Norte (Central Argentina) and their implications in prehispanic subsistence practices. Journal of Archaeological Science Reports 8: 426-433.

Macario, K. D., Alves, E. Q., Carvalho, C., Oliveira, F. M., Bronk Ramsey, C. Chivall, D. Souza, R. C., Simone, L. y D. C. Cavallari

2016 The use of terrestrial snails of the genera Megalobulimus and Thaumastus as representatives of the atmospheric carbon reservoir. Scientifics Reports 6: 1-7.

Marcellino, A. J., Berberián, E.E. y J. A. Pérez

1967 El yacimiento arqueológico de Los Molinos (Departamento Calamuchita, Córdoba). Publicaciones del Instituto de Antropología 26: 1-68.

Martín de Zurita, J.A.

1997 MS. Prospección y relevamiento de sitios. Informe elevado a la Dirección de Patrimonio Cultural de la Provincia de Córdoba.

Martínez Soler, B.

1958-59 Conchyliologia Ethnologica. El uso ornamental y ceremonial de algunas especies de moluscos. Runa X (1-2): 267-322.

Menghin, O. y A. R. González

1954 Excavaciones arqueológicas en el yacimiento de Ongamira, Córdoba, República Argentina. Notas del Museo de La Plata 17(67): 213-274.

Nimo, A.

1946 Arqueología de Laguna Honda (Yucat, Provincia de Córdoba). Publicaciones del Instituto de Arqueología, Lingüistica y Folklore "Dr. Pablo Cabrera" 16: 977-1045.

Nordenskiöld, E.

1903 [1993] Lugares precolombinos de asentamiento y entierro en la frontera sudoeste del Chaco. Traducción del original en sueco de 1903 por A. Fernández Distel y A. Distel. Serie Jujuy en el pasado, Universidad Nacional de Jujuy, San Salvador de Jujuy.

Ortiz, G. y N. Vargas Rodríguez

2015 Más allá del artefacto. Aproximación al ambiente y estrategias de uso de moluscos en la región del valle de San Francisco, Jujuy (0-500 D.C.). En Arqueomalacología. Abordajes metodológicos y casos de estudio en el Cono Sur, H. Hammondy M. Zubimendi (eds), pp. 59-77. Fundación de Historia Natural Félix de Azara. Buenos Aires.

Outes, F.

1911 Los tiempos prehistóricos y protohistóricos en la provincia de Córdoba. Revista del Museo de La Plata 17(4): 261-374.

Painceyra, R. y R. Martino

2004 Análisis de la estructura frágil en la zona de Cerro Colorado, Sierra Norte de Córdoba. Asociación Geológica Argentina, Serie D, Publicación especial 7: 107-111. 
Pastor, S.

2007 "Juntas y cazaderos". Las actividades grupales y la reproducción de las sociedades prehispánicas de las Sierras Centrales de Argentina. En Procesos sociales prehispánicos, A. E. Nielsen, M. C. Rivolta, V. Seldes, M. M. Vázquez y P. H. Mercolli (eds), pp. 361-376. Editorial Brujas, Córdoba.

2015 Acerca de la constitución de agentes sociales, objetos y paisajes. Una mirada desde las infraestructuras de molienda (Sierras de Córdoba, Argentina). En Condiciones de posibilidad de la reproducción social en sociedades prehispánicas y coloniales tempranas en las sierras pampeanas (República Argentina), J. Salazar (ed), pp. 402-441. Centro de Estudios Históricos "Profesor S. A. Segreti", Córdoba.

Pastor, S., Gordillo, S. y L. E. Tissera

2017a Objetos y paisajes multisensoriales del Holoceno Tardío inicial en el centro de Argentina (ca. 3900 AP). Acerca de un contexto arqueomalacológico de las Sierras de Córdoba. Intersecciones en Antropología 18: 317-327.

Pastor, S., Rivero, D. E., Recalde, M. A., Díaz, I. y G. Truyol

2017b Procesos y paisajes sociales en las sierras Centrales de Argentina durante el Holoceno tardío inicial (4200-2000 AP). Relaciones de la Sociedad Argentina de Antropología 42(2): 281-303.

Pedersen, A.

1953-54 El infrarrojo y su aplicación en la investigación de pinturas rupestres. Runa 6(12): 216-219.

Recalde, M. A.

2015 Representaciones en contexto. Características del paisaje rupestre de Cerro Colorado (Sierras del Norte, Córdoba, Argentina). Relaciones de la Sociedad Argentina de Antropología 40(2): 523-548.

Recalde, M. A. y L. López

2017 Las sociedades prehispánicas tardías en la región septentrional del Centro de Argentina (Sierras del Norte, Córdoba). Avances a su conocimiento desde los recursos vegetales. Chungara 49(4): 573-588.

Recalde, M. A. y E. Colqui

Representaciones rupestres de animales en Cerro Colorado (Sierras del Norte, centro de Argentina) y su papel en la construcción de identidades durante el Prehispánico tardío (ca. 1500-450 AP). Boletín del Museo Chileno de Arte Precolombino (En prensa).

Rumi, A., D. E. Gutiérrez Gregoric, V. Nuñez y Darrigran G.

2008 Malacología Latinoamericana. Moluscos de agua dulce de Argentina. Revista Biología Tropical 56 (1): 77-111.

Rivero, D. E.

2015 El surgimiento de la desigualdad social en la prehistoria de las Sierras de Córdoba (República Argentina). En Condiciones de posibilidad de la reproducción social en sociedades prehispánicas y coloniales tempranas en las Sierras Pampeanas (República Argentina), J. Salazar ed), pp. 15-40. CEH-CONICET, Córdoba. 
Serrano, A.

1945 Los Comechingones. Instituto de Arqueología, Lingüística y Folklore de la Universidad Nacional de Córdoba, Córdoba.

Strelin, J.

1995 Geomorfología de Cerro Colorado. Factores Ecológicos de Comportamiento 19(6): 4169.

Yanes, Y., Izeta, A.D., Cattáneo, R., Costa, T. y S., Gordillo

2014 Holocene paleoenvironmental $(\sim 4.5-1.7$ cal. kyr BP) conditions in central Argentina inferred from entire-shell and intra-shell stable isotope composition of land snails. The Holocene 24 (10): 1193-1205. 